\title{
A Novel Algorithm for the Adaptation of the Pole of Laguerre Filters
}

\author{
Christos Boukis, Danilo P. Mandic, Anthony G. Constantinides, and Lazaros C. Polymenakos
}

\begin{abstract}
This letter proposes a novel stochastic gradient algorithm for the online adaptation of the pole position in Laguerre filters. The proposed algorithm exploits the inherent relationship between the values of the filter coefficients and the value of the Laguerre pole. This leads to an unbiased solution and, hence, a more accurate estimate of the error gradient. Simulations in a system identification setting support the analysis.
\end{abstract}

Index Terms-Adaptive signal processing, orthonormal functions.

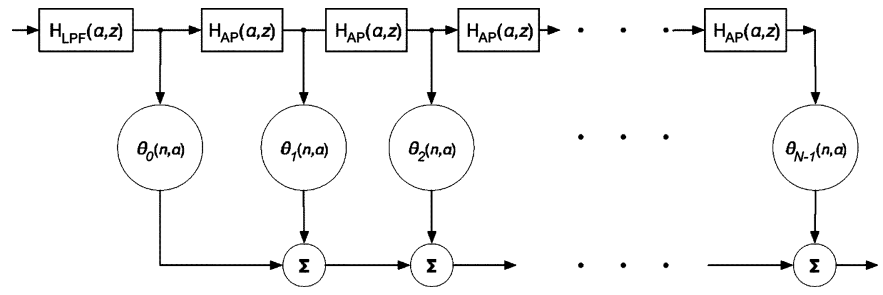

Fig. 1. A Laguerre filter of order $N-1$.

is given by

$$
y(n, \alpha)=\boldsymbol{\theta}^{T}(n, \alpha) \phi(n, \alpha)=\sum_{i=0}^{N-1} \theta_{i}(n, \alpha) l_{i}(n, \alpha) \otimes u(n)
$$

where the symbol $\otimes$ denotes convolution and $\boldsymbol{\theta}=$ $\left[\theta_{0}(n, \alpha), \ldots, \theta_{N-1}(n, \alpha)\right]^{T}$ the filter weights vector. The elements $\phi_{m}(n, \alpha)$ of the regressor vector $\phi(n, \alpha)=$ $\left[\phi_{0}(n, \alpha), \ldots, \phi_{N-1}(n, \alpha)\right]^{T}$ are computed from the recursions

$$
\begin{aligned}
\phi_{0}(n, \alpha)= & \alpha \phi_{0}(n-1, \alpha)+\sqrt{1-\alpha^{2}} u(n) \\
\phi_{1}(n, \alpha)= & \alpha \phi_{1}(n-1, \alpha)+\phi_{0}(n-1, \alpha)-\alpha \phi_{0}(n, \alpha) \\
\vdots \quad & \vdots \\
\phi_{N-1}(n, \alpha)= & \alpha \phi_{N-1}(n-1, \alpha)+\phi_{N-2}(n-1, \alpha) \\
& -\alpha \phi_{N-2}(n, \alpha) .
\end{aligned}
$$

These can be rewritten in its vector-matrix form to yield

$$
\boldsymbol{A} \boldsymbol{\phi}(n, \alpha)=\boldsymbol{B} \boldsymbol{\phi}(n-1, \alpha)+\boldsymbol{c} u(n)
$$

where $\boldsymbol{A}=\boldsymbol{I}+\alpha \boldsymbol{I}_{-}, \boldsymbol{B}=\alpha \boldsymbol{I}+\boldsymbol{I}_{-}, \boldsymbol{c}=\left[\sqrt{1-\alpha^{2}}, 0, \cdots, 0\right]^{T}$, $\boldsymbol{I}$ the $N \times N$ unitary matrix, and $\boldsymbol{I}_{-}$an $N \times N$ matrix whose elements are zero apart from those of its sub-diagonal, which are equal to unity. A compact recursive form for the computation of the regressor vector can now be expressed as

$$
\boldsymbol{\phi}(n, \alpha)=\boldsymbol{A}^{-1} \boldsymbol{B} \boldsymbol{\phi}(n-1, \alpha)+\boldsymbol{A}^{-1} \boldsymbol{c} u(n) .
$$

The inverse of the matrix $A$ is an $N \times N$ lower triangular matrix whose elements $a_{i j}^{(i n v)}$ are given by

$$
a_{i j}^{(i n v)}= \begin{cases}0, & \text { for } i>j \\ (-\alpha)^{(j-i)}, & \text { for } i \leq j\end{cases}
$$

sociate editor coordinating the review of this manuscript and approving it for publication was Dr. Stefano Galli.

C. Boukis and L. C. Polymenakos are with the Athens Information Technology, Peania/Athens 19002, Greece (e-mail: christos.boukis@ait.edu.gr; lcp@ait.edu.gr).

D. P. Mandic and A. G. Constantinides are with Imperial College, London SW7 2BT, U.K. (e-mail: d.mandic@imperial.ac.uk; agc@imperial.ac.uk).

Digital Object Identifier 10.1109/LSP.2006.873140

\section{A. Coefficient Updating}

Since the output of a Laguerre filter depends linearly on its weights, techniques used for the update of FIR filter coefficients 
can be adopted. Thus, applying a steepest descent scheme that minimizes the square of the instantaneous output error of such a filter results in the Laguerre least mean-square (LLMS) algorithm, given by

$$
\boldsymbol{\theta}(n+1, \alpha)=\boldsymbol{\theta}(n, \alpha)+\mu_{\theta} e(n, \alpha) \boldsymbol{\phi}(n, \alpha)
$$

where $\mu_{\theta}$ is a time invariant step size. Given the desired response $d(n)$, the output error that drives the adaptation in (3) can be expressed as

$$
e(n, \alpha)=d(n)-\boldsymbol{\theta}^{T}(n, \alpha) \phi(n, \alpha) .
$$

Convergence of this algorithm is ensured if the step size is smaller than the inverses of the eigenvalues of the autocorrelation matrix $\boldsymbol{R}_{\phi}(\alpha)=E\left\{\boldsymbol{\phi}(n, \alpha) \boldsymbol{\phi}^{T}(n, \alpha)\right\}$. Finally, the Wiener solution can be found as [5]

$$
\boldsymbol{\theta}(\alpha)=\boldsymbol{R}_{\phi}^{-1}(\alpha) \boldsymbol{r}_{d \phi}(\alpha)
$$

where $\boldsymbol{r}_{d \phi}(\alpha)=E\{d(n) \boldsymbol{\phi}(n, \alpha)\}$ is the cross-correlation matrix.

The performance of an adaptive Laguerre filter depends critically on the value of its pole $\alpha$. In particular, from equation (5), it is clear that for a given filter order $(N-1)$, the steady-state error and bias due to truncation are a function of $\alpha$.

For a filter of finite order $(N-1)$, the optimal Laguerre pole $\alpha^{\star}$ is the one that minimizes the steady-state MSE given by

$$
\mathcal{E}_{\min }(\alpha)=\frac{1}{2}\left(E\left\{d^{2}(n)\right\}-\boldsymbol{r}_{d \phi}^{T}(\alpha) \boldsymbol{R}_{\phi}^{-1}(\alpha) \boldsymbol{r}_{d \phi}(\alpha)\right) .
$$

Setting the derivative of $\mathcal{E}_{\min }(\alpha)$, from (6) with respect to $\alpha$ to zero, will result in a set of equations whose solution yields the optimal Laguerre pole $\alpha^{\star}$. These equations though are usually nonlinear, and solving them is not trivial. Alternatively, the optimal pole value can be found heuristically [6] based on the condition $\theta_{N-1}\left(\alpha^{\star}\right) \theta_{N}\left(\alpha^{\star}\right)=0$ that holds for the optimal pole of a truncated Laguerre expansion of $\operatorname{order} N-1$.

\section{B. Previous Algorithm [3]}

Computing the pole $\alpha^{\star}$ either heuristically or directly is a computationally demanding task. Furthermore, in nonstationary environments where $\alpha^{\star}$ is time-varying, its continuous computation is required. These obstacles can be bypassed with the employment of adaptive systems that estimate $\alpha^{\star}$ recursively [3], [4].

In [3], a stochastic gradient descent technique that minimizes the square of the instantaneous output error for the optimization of the pole of Gamma filters was proposed. Applying this algorithm for the adaptation of the pole of Laguerre filters results in the following recursive equation:

$$
\alpha(n+1)=\alpha(n)+\mu_{\alpha} e(n, \alpha) \gamma(n, \alpha)
$$

where $\mu_{\alpha}$ is the learning rate for the updating of the adaptive pole $\alpha(n+1)$. The term $\gamma(n, \alpha)$ is the gradient of the output signal with respect to the pole $\alpha(n)$, and it is given by

$$
\gamma(n, \alpha)=\boldsymbol{\theta}^{T}(n, \alpha) \frac{d \boldsymbol{\phi}(n, \alpha)}{d \alpha(n)}
$$

The gradient $d \boldsymbol{\phi}(n, \alpha) / d \alpha(n)$ can be found by differentiating both sides of (1) with respect to the pole $\alpha(n)$ to be

$$
\begin{aligned}
\frac{d \boldsymbol{\phi}(n, \alpha)}{d \alpha(n)}= & \boldsymbol{A}^{-1}(n) \boldsymbol{B}(n) \frac{d \boldsymbol{\phi}(n-1, \alpha)}{d \alpha(n-1)} \\
& +\left[\mathbb{D}_{A}(n) \boldsymbol{B}(n)+\boldsymbol{A}^{-1}(n)\right] \boldsymbol{\phi}(n-1, \alpha) \\
& +\left[\mathbb{D}_{A}(n) \boldsymbol{c}(n)+\boldsymbol{A}^{-1}(n) \mathbb{D}_{c}(n)\right] u(n)
\end{aligned}
$$

where the $N \times N$ matrix $\mathbb{D}_{A}(n)$ and the $N \times 1$ vector $\mathbb{D}_{c}(n)$ can be derived by differentiating the matrix $\boldsymbol{A}^{-1}$ and the vector $c$ that are present in the recursive equation (1) with respect to $\alpha(n)$. Notice that the adaptation of the pole is performed in parallel with the adaptation of the coefficients, as indicated by (3). Hereafter, this algorithm will be referred as the "simplified gradient adaptive pole (SGAP) algorithm."

\section{Proposed Algorithm}

In the derivation of the SGAP algorithm in [3], the dependence of the coefficients on the pole of the filter was neglected, and the gradient of the output with respect to the pole of the filter was approximated by (8). The gradient vector $d \boldsymbol{\theta}(n) / d \alpha(n)$ though is nonzero, since both the coefficient vector $\boldsymbol{\theta}(n, \alpha)$ and the current Laguerre pole $\alpha(n)$ depend on previous values of $\alpha(n)$. Thus

$\gamma(n, \alpha)=\frac{d y(n, \alpha)}{d \alpha(n)}=\frac{d \boldsymbol{\theta}^{T}(n, \alpha)}{d \alpha(n)} \boldsymbol{\phi}(n, \alpha)+\boldsymbol{\theta}^{T}(n, \alpha) \frac{d \boldsymbol{\phi}(n, \alpha)}{d \alpha(n)}$.

Differentiating both sides of (3) with respect to $\alpha(n)$, and assuming slow pole convergence, i.e., $\alpha(n) \approx \alpha(n-1)$, which results in $d \boldsymbol{\phi}(n-1, \alpha) / d \alpha(n) \approx d \boldsymbol{\phi}(n-1, \alpha) / d \alpha(n-1)$ [7], yields

$$
\begin{aligned}
\frac{d \boldsymbol{\theta}(n, \alpha)}{d \alpha(n)}= & {\left[\boldsymbol{I}-\mu_{\theta} \boldsymbol{\phi}(n-1, \alpha) \boldsymbol{\phi}^{T}(n-1, \alpha)\right] \frac{d \boldsymbol{\theta}(n-1, \alpha)}{d \alpha(n-1)} } \\
& +\mu_{\theta}\left[e(n-1) \boldsymbol{I}-\boldsymbol{\phi}(n-1, \alpha) \boldsymbol{\theta}^{T}(n-1, \alpha)\right] \\
& \times \frac{d \boldsymbol{\phi}(n-1, \alpha)}{d \alpha(n-1)} .
\end{aligned}
$$

Equations (3), (4), (7), (10), and (11) consist the proposed full gradient adaptive pole (FGAP) algorithm (see Table I). Contrary to most of the existing techniques [3], [4], this algorithm takes into account the dependence of its coefficients on the parameter $\alpha(n)$ and thus yields more accurate estimates.

\section{Computational Complexity Analysis}

For the updating of the value of the pole with the FGAP algorithm, $2 N^{3}+10 N^{2}+8 N+3$ multiplications and $2 N^{3}+$ $7 N^{2}-2 N$ additions are required (see Table I) at every iteration. In this analysis, the computation of the inverse of the matrix $A$ is not included. This operation though is not demanding since it is performed from (2) with substitution of the current pole value $\alpha(n)$, and thus, only $N-2$ multiplications are required. The computation of the matrix $\mathbb{D}_{A}$ and the vector $\mathbb{D}_{c}$ can be performed from analytic expressions as well, with $\mathcal{O}(N)$ multiplications and $\mathcal{O}(N)$ additions at every iteration. Notice that, since 
TABLE I

FGAP Algorithm. DePendence of the MAtrices $\boldsymbol{A}, \boldsymbol{B}$, AND $\mathbb{D}_{A}$ AND THE VECTORS $\boldsymbol{c}$ AND $\mathbb{D}_{c}$ ON THE LAGUERRE $\alpha$ POLE, AND THE TIME INDEX $n$ IS DELIBERATELY OMITTED

\begin{tabular}{|c|c|c|}
\hline Definitions & \multicolumn{2}{|c|}{ Initial Conditions } \\
\hline $\boldsymbol{\theta}(n)=\left[\theta_{0}(n), \theta_{1}(n), \ldots, \theta_{N-1}(n)\right]^{T}$ & \multicolumn{2}{|c|}{$\overline{\boldsymbol{\theta}(0)=[0,0, \ldots, 0]^{T}}$} \\
\hline$\phi(n)=\left[\phi_{0}(n), \phi_{1}(n), \ldots, \phi_{N-1}(n)\right]^{T}$ & \multicolumn{2}{|c|}{$\phi(0)=[0,0, \ldots, 0]^{T}$} \\
\hline $\boldsymbol{\xi}(n)=d \boldsymbol{\theta}(n) / d \alpha(n)$ & \multicolumn{2}{|c|}{$\boldsymbol{\xi}(0)=[0,0, \ldots, 0]^{T}$} \\
\hline $\boldsymbol{\tau}(n)=d \phi(n) / d \alpha(n)$ & \multicolumn{2}{|c|}{$\boldsymbol{\tau}(0)=[0,0, \ldots, 0]^{T}$} \\
\hline$\gamma(n)=d y(n) / d \alpha(n)$ & \multicolumn{2}{|l|}{$\gamma(0)=0$} \\
\hline Algorithm & Mul & Add \\
\hline $\boldsymbol{P}_{\mathbf{1}}(n)=\boldsymbol{A}^{-1} \boldsymbol{B}$ & $\overline{N^{3}}$ & $\overline{N^{3}-N^{2}}$ \\
\hline$q_{1}(n)=A^{-1} c$ & $N^{2}$ & $N^{2}-N$ \\
\hline $\boldsymbol{P}_{2}(n)=\left[\mathbb{D}_{A} \boldsymbol{B}+\boldsymbol{A}^{-1}\right]$ & $N^{3}$ & $N^{3}$ \\
\hline $\boldsymbol{q}_{2}(n)=\left[\mathbb{D}_{A} \boldsymbol{c}+\boldsymbol{A}^{-1} \mathbb{D}_{c}\right]$ & $2 N^{2}$ & $2 N^{2}-2 N$ \\
\hline$\phi(n)=\boldsymbol{P}_{\mathbf{1}}(n) \phi(n-1)+\boldsymbol{q}_{\mathbf{1}}(n) u(n)$ & $N^{2}+N$ & $N^{2}-N$ \\
\hline$e(n)=d(n)-\boldsymbol{\theta}^{T}(n) \phi(n)$ & $N$ & $N$ \\
\hline $\boldsymbol{\theta}(n+1)=\boldsymbol{\theta}(n)+\mu e(n) \boldsymbol{\phi}(n)$ & $N+1$ & $N$ \\
\hline $\begin{array}{l}\boldsymbol{\tau}(n)=\boldsymbol{P}_{\mathbf{1}}(n) \boldsymbol{\tau}(n-1)+\boldsymbol{P}_{\mathbf{2}}(n) \boldsymbol{\phi}(n-1) \\
+\boldsymbol{q}_{\mathbf{2}}(n) u(n)\end{array}$ & $2 N^{2}+N$ & $2 N^{2}-2 N$ \\
\hline $\boldsymbol{F}_{1}(n)=\left[\boldsymbol{I}-\mu \boldsymbol{\phi}(n-1) \boldsymbol{\phi}^{T}(n-1)\right]$ & $N^{2}+N$ & $N$ \\
\hline $\left.\boldsymbol{F}_{2}(n)=e(n-1) \boldsymbol{I}-\boldsymbol{\phi}(n-1) \boldsymbol{\theta}^{T}(n-1)\right]$ & $N^{2}$ & $N$ \\
\hline $\begin{array}{l}\boldsymbol{\xi}(n)=\boldsymbol{F}_{1}(n) \boldsymbol{\xi}(n-1) \\
+\mu \boldsymbol{F}_{2}(n) \boldsymbol{\tau}(n-1)\end{array}$ & $\begin{array}{c}2 N^{2}+N \\
+1\end{array}$ & $2 N^{2}-2 N$ \\
\hline$\gamma(n)=\boldsymbol{\xi}^{T}(n) \boldsymbol{\phi}(n)+\boldsymbol{\theta}^{T}(n) \boldsymbol{\tau}(n)$ & $2 N$ & $2 N-1$ \\
\hline$\alpha(n+1)=\alpha(n)+\rho e(n) \gamma(n)$ & 2 & \\
\hline
\end{tabular}

SGAP algorithm neglects the dependence of the coefficients of the filter on the value of its pole and thus does not compute $\boldsymbol{\tau}(n)$, it requires $4 N^{2}+2 N+1$ fewer multiplications and $N^{2}$ fewer additions than the proposed FGAP algorithm.

Exploiting the fundamental property of Laguerre filters [6], given by

$$
\frac{d L_{m}(z, \alpha)}{d \alpha}=\frac{(m+1) L_{m+1}(z, \alpha)-m L_{m-1}(z, \alpha)}{1-\alpha^{2}}
$$

a significant relaxation of the computational requirements, which applies to both algorithms, can be achieved. Indeed, due to the fact that the input $u(n)$ and the Laguerre pole $\alpha$ are uncoupled, multiplying both sides of (12) by $U(z)$ and taking the inverse $\mathcal{Z}$ transform results in

$$
\frac{d \phi_{m}(n, \alpha)}{d \alpha(n)}=\frac{(m+1) \phi_{m+1}(n, \alpha)-m \phi_{m-1}(n, \alpha)}{1-\alpha^{2}}
$$

where $m=0,1, \ldots, N-1$. Using (13) instead of (9) in the update of the adaptive pole reduces the computational complexity of the FGAP algorithm by $N^{3}+4 N^{2}$ multiplications and $N^{3}+4 N^{2}-4 N$ additions. Notice that using (13) instead of (9) does not affect the performance of the algorithm in terms of steady-state MSE and convergence speed.

Further reduction of the computational burden can be achieved by observing that only the elements of the diagonal and the sub-diagonal of the matrices $A$ and $B$ are nonzero and that $c_{i}=0$ for $i \neq 0$. This implies that the matrix products

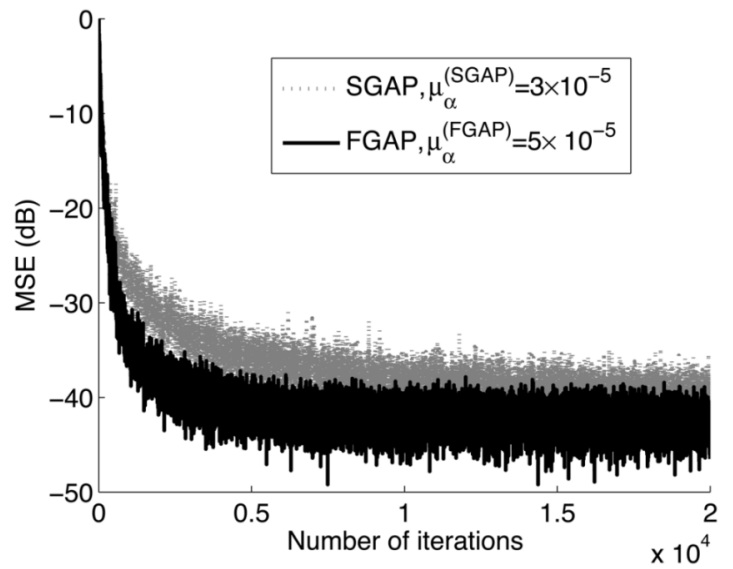

Fig. 2. Comparison of the MSE curves of the SGAP (grey dotted line) and the FGAP (black solid line) algorithms.

$A^{-1} \boldsymbol{B}, A^{-1} \boldsymbol{c}, \mathbb{D}_{A} \boldsymbol{B}, \mathbb{D}_{A} \boldsymbol{c}$, and $\boldsymbol{A}^{-1} \mathbb{D}_{c}$ can be computed within $\mathcal{O}(N)$ multiplications and $\mathcal{O}(N)$ additions.

\section{Simulation Results}

The simulations that were carried out intended to illustrate the effect of the gradient of the weight vector with respect to the Laguerre pole on the convergence behavior of the adaptive pole. To this cause, the derived FGAP algorithm was compared to the SGAP algorithm [3] that neglects the dependence of the coefficients on the pole. Both algorithms were employed for the adaptation of the pole of a Laguerre filter of order 9. The application was a typical system identification problem where the transfer function of unknown channel was ${ }^{1}$ as in (14), shown at the bottom of the page.

In Fig. 2, the learning curve of the SGAP algorithm (black dotted line) is presented along with that of the FGAP algorithm (grey solid line). The learning rate of the pole adaptation was $\mu_{\alpha}^{(\mathrm{SGAP})}=3 \times 10^{-5}$ for the SGAP and $\mu_{\alpha}^{(\mathrm{FGAP})}=5 \times 10^{-5}$ for the FGAP algorithm. This graph was derived by averaging the results of a Monte Carlo experiment consisting of 20 independent simulation runs. It is observed that the steady-state MSE of the FGAP is lower than that of the SGAP, which implies more accurate modeling of the unknown channel. Notice that for $\mu_{\alpha}^{(\mathrm{SGAP})} \geq 3 \times 10^{-5}$, the SGAP algorithm was often diverging. Hence, catering for the dependence of the coefficients on the pole results in a wider range of allowable values for the learning rate of the adaptive pole. Both algorithms employed a step size $\mu_{\theta}=3 \times 10^{-2}$ for the adaptation of the filter coefficients.

A comparison of the trajectories of the adaptive pole of a Laguerre filter derived from the application of the FGAP (black lines) and the SGAP (grey lines) algorithms is provided in Fig. 3

${ }^{1}$ Notice that all the poles were real valued, since Laguerre filters cannot model accurately channels with conjugate pairs of complex poles, that is, systems with strong resonances. For that case, Kautz filters are more appropriate.

$$
H(z)=\frac{1.0000-1.44 z^{-1}+0.374 z^{-2}}{1+1.5301 z^{-1}+0.532 z^{-2}-0.0549 z^{-3}-0.0195 z^{-4}+0.0014 z^{-5}}
$$




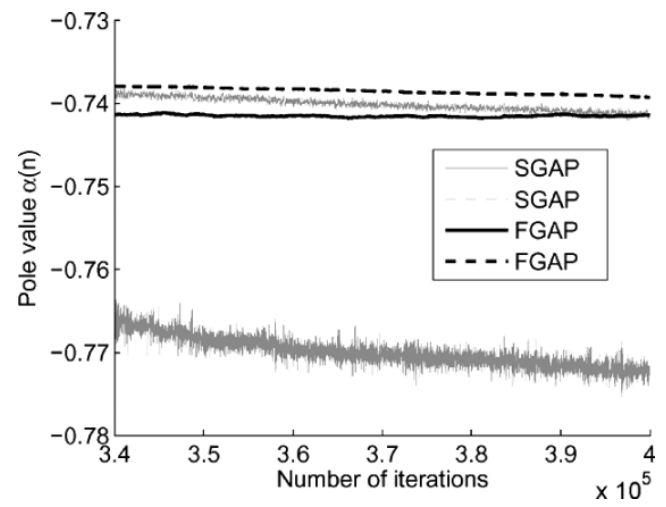

Fig. 3. Comparison of the trajectories of poles adapted with the SGAP (grey lines) and the FGAP (black lines) algorithms.

for two values of the learning rate $\mu_{\alpha}=3 \times 10^{-5}$ (dashed lines) and $\mu_{\alpha}=5 \times 10^{-5}$ (solid lines). From this plot, it is observed that when the FGAP algorithm is employed, the steady-state value of the adaptive pole is independent of the value of the learning rate $\mu_{\alpha}$, which is not the case for SGAP. This can be explained as follows: the gradient of the output error with respect to the varying pole contains two fundamental terms: the gradient of the regressor vector and the gradient of the filter weights vector with respect to the Laguerre pole. At steady state, these terms cancel each other like two forces with equal magnitude and opposite direction that are applied to the same point. In the absence of one of these two gradients though, the pole cannot remain in steady state, even when this is reached due to the nonzero overall gradient term. Indeed, when SGAP is used, the pole value changes constantly. Moreover, the pole trajectories of the SGAP are noisy, which is not the case for the FGAP. Finally, notice that all the pole trajectories are dominated by two trends: fast convergence to the vicinity of the steady-state value initially and very slow convergence to the exact steady-state solution afterward.

Fig. 4 represents the steady-state MSE of a Laguerre filter of length 10 with fixed pole that is employed for the identification of the previously given unknown channel. From this and Fig. 3, it is observed that the steady-state value of the adaptive pole when the FGAP algorithm is employed coincides with the optimum pole value. The computation of the steady-state MSE for a given pole value was performed by averaging the last 1000 values of a 3000-samples-long MSE curve, which was derived from a Monte Carlo experiment consisting of 20 independent simulation runs. The normalized LMS algorithm with step size 1 was used for the adaptation of the filter coefficients. The input signal was white noise of zero mean and unitary variance. Notice that under these circumstances, steady state is reached within a few hundred iterations.

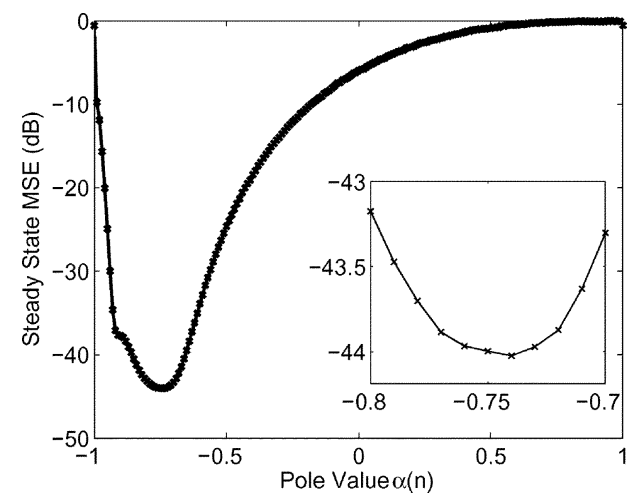

Fig. 4. Steady-state MSE of a tenth-order Laguerre filter as a function of the value of its pole.

\section{CONCLUSION}

A novel approach for the adaptation of the pole of a Laguerre filter has been introduced. This has been achieved by a steepest descent type of optimization applied to the filter parameters. It has been shown that, unlike the existing approaches, this algorithm does converge to an unbiased solution. This is due to the fact that the proposed approach caters for dependence of the filter output on the value of the Laguerre pole. The approach is generic, and its extensions to other orthonormal basis filters are straightforward. The performance of the proposed algorithm has been verified through simulations in a system identification setting.

\section{ACKNOWLEDGMENT}

The authors would like to thank the anonymous reviewers for their thorough and insightful comments, which have been extremely helpful toward the final version of this manuscript.

\section{REFERENCES}

[1] B. Wahlberg, "System identification using Laguerre models," IEEE Trans. Autom. Control, vol. 36, no. 5, pp. 551-562, May 1991.

[2] N. Tanguy, P. Vilbé, and L. C. Calvez, "Optimum choice of free parameter in orthonormal approximations," IEEE Trans. Autom. Control, vol. 40, no. 10, pp. 1811-1813, Oct. 1995.

[3] J. Principe, B. de Vries, and P. de Oliveira, "The gamma-filter-a new class of adaptive IIR filters with restricted feedback," IEEE Trans. Signal Process., vol. 41, no. 2, pp. 649-656, Feb. 1993.

[4] H. Belt and A. den Brinker, "Laguerre filters with adaptive pole optimization," in Proc. IEEE Int. Symp. Circuits Systems, 1996, vol. 2, pp. $37-40$.

[5] J. Proakis, C. Rader, F. Ling, and C. Nikias, Advanced Digital Signal Processing. New York: Macmillan, 1992.

[6] T. Oliveira e Silva, "On the determination of the optimal pole position of Laguerre filters," IEEE Trans. Signal Process., vol. 43, no. 9, pp. 2079-2087, Sep. 1995.

[7] P. Regalia, Adaptive IIR Filtering in Signal Processing and Control. New York: Marcel Decker, 1995. 